\title{
Correction to: Predictive value of positron emission tomography for the prognosis of immune checkpoint inhibitors (ICls) in malignant tumors
}

\author{
Qing Wu ${ }^{1}$. Junjin Liu ${ }^{1} \cdot$ Yanhong Zhang ${ }^{1}$. Sumei Wu ${ }^{1} \cdot$ Xianhe Xie $^{1} \mathbb{D}$
}

Published online: 11 March 2020

(c) Springer-Verlag GmbH Germany, part of Springer Nature 2020

\section{Correction to: Cancer Immunology, Immunotherapy https://doi.org/10.1007/s00262-020-02515-w}

The original version of this article unfortunately contained a mistake. The correct information is given in the following.

In Abstract, the third sentence of the "Methods" section should read as:

The results were analyzed utilizing Stata 12.0 statistical software.

In the "Materials and methods" section, the first sentence of the "Statistical methods" section should read as:

Statistical analyses were performed with Stata 12.0 statistical software.
Publisher's Note Springer Nature remains neutral with regard to jurisdictional claims in published maps and institutional affiliations.

The original article can be found online at https://doi.org/10.1007/ s00262-020-02515-w.

Xianhe Xie

xiexianhe@ jmu.edu.cn

1 Department of Oncology, The First Affiliated Hospital of Fujian Medical University, Chazhong Road No. 20, Fuzhou 350005, Fujian, China 